\title{
The New Kumaraswamy Kumaraswamy Weibull Distribution with Application
}

\author{
Mahmoud R. Mahmoud \\ Institute of Statistical Studies \& Research, Cairo University, Egypt \\ mrmahmoud@issr.cu.edu.eg \\ El-Sayed A. El-Sherpieny \\ Institute of Statistical Studies \& Research, Cairo University, Egypt \\ ahmedc55@yahoo.com \\ Mohamed A. Ahmed \\ Institute of Statistical Studies \& Research, Cairo University, Egypt \\ mrmohamedali2005@yahoo.com
}

\begin{abstract}
The Weibull distribution has been used over the past decades for modeling data in many fields so finding generalization of the Weibull distribution becomes very useful to fit more cases or get better fits than before. In this paper, the Kumaraswamy Kumaraswamy Weibull ( $\mathrm{Kw} \mathrm{Kw} \mathrm{W)} \mathrm{distribution} \mathrm{is} \mathrm{presented} \mathrm{for}$ the first time and we show that it generalizes many important distributions. The probability density function (pdf), the cumulative distribution function (cdf), moments, quantiles, the median, the mode, the mean deviation, the entropy, order statistics, L-moments, extreme value and parameters estimation based on maximum likelihood are obtained for the $\mathrm{Kw} \mathrm{Kw} \mathrm{W} \mathrm{distribution.} \mathrm{An} \mathrm{illustration} \mathrm{study} \mathrm{and} \mathrm{a} \mathrm{real} \mathrm{data} \mathrm{set}$ are used to illustrate the potentiality and application of the new $\mathrm{Kw} \mathrm{Kw} \mathrm{W}$ distribution.
\end{abstract}

Keywords: The Weibull distribution, The Kumaraswamy Kumaraswamy family, Moments, Order statistics, L-moments, Entropy, Extreme values, Maximum likelihood estimation.

\section{Introduction}

Eugene et al. (2002) presented for the first time the family of beta generalized distributions which has the following cdf and pdf

$$
F(x)=\frac{1}{B(a, b)} \int_{0}^{G(x ; W)} t^{a}(1-t)^{b} d t ; a, b>0 ; 0<t<1 ;-\infty<x<\infty
$$

and

$$
f(x)=\frac{1}{B(a, b)} g(x ; W) G(x ; W)^{a-1}\{1-G(x ; W)\}^{b-1} ; a, b>0 ;-\infty<x<\infty
$$

Where $G(x ; W)$ and $g(x ; W)$ are the cdf and the pdf of the baseline distribution, $\mathrm{W}$ is parameters vector of the baseline distributions. 
Wahed (2006) presented a general method of constructing extended families of distribution from an existing continuous class using the following equation

$$
F(x ; T, W)=\int_{0}^{G_{1}(x, W)} g_{2}(\mathrm{t} ; \mathrm{T}) d t ; 0<t<1
$$

Where $G_{1}(x ; W)$ and $g_{1}(x ; W)$ are the cdf and pdf of the baseline distribution, $G_{2}(t ; T)$ and $g_{2}(t ; T)$ are the cdf and the pdf of the generator distribution, $\mathrm{T}$ and $\mathrm{W}$ are respectively parameters vectors of generator and baseline distributions.

Cordeiroa and Castro (2010) presented the family of Kumaraswamy generalized distributions which has the following cdf and pdf

$$
F(x ; \mathrm{a}, \mathrm{b}, W)=1-\left\{1-G(x ; W)^{a}\right\}^{b} ; a, b>0 ;-\infty<x<\infty
$$

and

$$
f(x ; \mathrm{a}, \mathrm{b}, W)=a b g(x) G(x)^{a-1}\left\{1-G(x)^{a}\right\}^{b-1} ; a, b>0 ;-\infty<x<\infty
$$

Where $G(x ; W)$ and $g(x ; W)$ are the cdf and the pdf of the baseline distribution, $\mathrm{W}$ is parameters vector of the baseline distributions.

Mahmoud et al. (2015) presented for the first time the Kumaraswamy Kumaraswamy family of generalized distributions which has the following cdf and pdf

$$
F(x ; a, b, \alpha, \beta, \underline{\mathrm{W}})=1-\left\{1-\left[1-\left(1-G^{\alpha}(x, \underline{\mathrm{W}})\right)^{\beta}\right]^{a}\right\}^{b} ;-\infty<x<\infty ; a, b, \alpha, \beta>0
$$

and

$$
\begin{aligned}
f(x ; a, b, \alpha, \beta, \underline{\mathrm{W}}) & =a b \alpha \beta g(x, \underline{\mathrm{W}}) G^{\alpha-1}(x, \underline{\mathrm{W}})\left(1-G^{\alpha}(x, \underline{\mathrm{W}})\right)^{\beta-1}\left[1-\left(1-G^{\alpha}(x, \underline{\mathrm{W}})\right)^{\beta}\right]^{a-1} \\
& \times\left\{1-\left[1-\left(1-G^{\alpha}(x, \underline{\mathrm{W}})\right)^{\beta}\right]^{a}\right\}^{b-1} ;-\infty<x<\infty ; a, b, \alpha, \beta>0
\end{aligned}
$$

Where $G(x ; \underline{\mathrm{W}})$ and $g(x ; \underline{\mathrm{W}})$ are the cdf and the pdf of the baseline distribution, $\mathrm{W}$ is parameters vector of the baseline distributions.

The main idea of this paper is based on deriving the $\mathrm{Kw} \mathrm{Kw} W$ distribution from the Kumaraswamy Kumaraswamy family of generalized distributions see Mahmoud et al.(2015), where the generator distribution is the Kumaraswamy Kumaraswamy distribution, see El-Sherpieny and Ahmed (2014), which has the following cdf

$$
G(x)=1-\left(1-x^{\alpha}\right)^{\beta} \quad ; 0<x<1 ; \alpha, \beta>0
$$




\section{The New Kumaraswamy Kumaraswamy Weibull Distribution}

Since the cdf and pdf of the Weibull distribution respectively are

$$
G(x ; \theta, \lambda)=1-e^{-(\lambda x)^{\theta}} ; x, \theta, \lambda>0
$$

and

$$
g(x ; \theta, \lambda)=\theta \lambda^{\theta} x^{\theta-1} e^{-(\lambda x)^{\theta}} ; x, \theta, \lambda>0
$$

Then, substituting (3) and (4) into (1) and (2) yields the cdf and the pdf of Kw Kw W distribution as follows

$$
F(x ; M)=1-\left\{1-\left[1-\left(1-\left(1-e^{-(\lambda x)^{\theta}}\right)^{\alpha}\right)^{\beta}\right]^{a}\right\}^{b} ; x>0 ; a, b, \alpha, \beta, \theta, \lambda>0
$$

and

$$
\begin{aligned}
f(x ; M) & =a b \alpha \beta \theta \lambda^{\theta} x^{\theta-1} e^{-(\lambda x)^{\theta}}\left(1-e^{-(\lambda x)^{\theta}}\right)^{\alpha-1}\left(1-\left(1-e^{-(\lambda x)^{\theta}}\right)^{\alpha}\right)^{\beta-1} \\
& \times\left[1-\left(1-\left(1-e^{-(\lambda x)^{\theta}}\right)^{\alpha}\right)^{\beta}\right]^{a-1}\left\{1-\left[1-\left(1-\left(1-e^{-(\lambda x)^{\theta}}\right)^{\alpha}\right)^{\beta}\right]^{a}\right\}^{b-1}
\end{aligned}
$$

Where $M=(a, b, \alpha, \beta, \theta, \lambda)$, the $\mathrm{Kw} \mathrm{Kw} \mathrm{W} \mathrm{distribution} \mathrm{has} \mathrm{five} \mathrm{shape} \mathrm{parameters} \mathrm{a}, \mathrm{b}$, $\alpha, \beta, \theta$ and has one scale parameter $\lambda$.

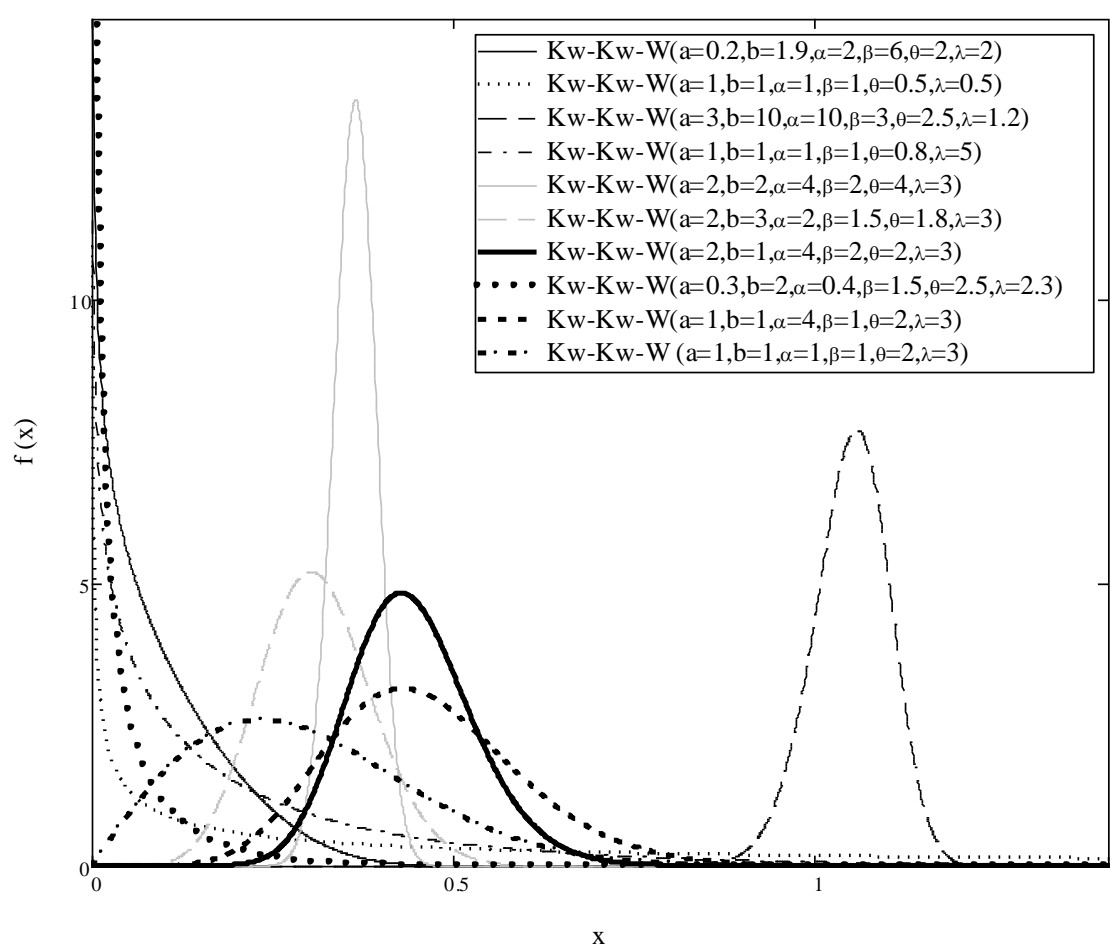

Figure 1: Plot of the $\mathrm{Kw} \mathrm{Kw} \mathrm{W}$ pdf for some parameter values

We see from the last Figure that the sex parameters give a high degree of flexibility for the $\mathrm{Kw} \mathrm{Kw} \mathrm{W}$ distribution. 
Some important sub-models of the $\mathrm{Kw} \mathrm{Kw} \mathrm{W} \mathrm{distribution} \mathrm{are} \mathrm{illustrated} \mathrm{in} \mathrm{the} \mathrm{following}$ table

\begin{tabular}{|l|l|c|c|c|c|c|c|}
\hline$\#$ & Distribution & $a$ & $b$ & $\alpha$ & $\beta$ & $\theta$ & $\lambda$ \\
\hline 1 & Kw Kw exponential & - & - & - & - & 1 & - \\
\hline 2 & Kw Kw Rayleigh & - & - & - & - & 2 & - \\
\hline 3 & Exponentiated Kw W & - & 1 & - & - & - & - \\
\hline 4 & Exponentiated Kw exponential & - & 1 & - & - & 1 & - \\
\hline 5 & Exponentiated Kw Rayleigh & - & 1 & - & - & 2 & - \\
\hline 6 & Exponentiated Generalized W & - & 1 & 1 & - & - & - \\
\hline 7 & Exponentiated Generalized exponential & - & 1 & 1 & - & 1 & - \\
\hline 8 & Exponentiated Generalized Rayleigh & - & 1 & 1 & - & 2 & - \\
\hline 9 & Kw W & 1 & 1 & - & - & - & - \\
\hline 10 & Kw exponential & 1 & 1 & - & - & 1 & - \\
\hline 11 & Kw Rayleigh & 1 & 1 & - & - & 2 & - \\
\hline 12 & Exponentiated W & 1 & 1 & - & 1 & - & - \\
\hline 13 & Exponentiated exponential & 1 & 1 & - & 1 & 1 & - \\
\hline 14 & Exponentiated Rayleigh & 1 & 1 & - & 1 & 2 & - \\
\hline 15 & W & 1 & 1 & 1 & 1 & - & - \\
\hline 16 & Exponential & 1 & 1 & 1 & 1 & 1 & - \\
\hline 17 & Rayleigh & 1 & 1 & 1 & 1 & 2 & - \\
\hline
\end{tabular}

\subsection{An Expansion for The pdf}

We shall obtain expansion for the pdf of the $\mathrm{Kw} \mathrm{Kw} \mathrm{W} \mathrm{distribution} \mathrm{as} \mathrm{follows:}$

Using the binomial expansion of the pdf of the $\mathrm{Kw} \mathrm{Kw} \mathrm{W}$ distribution, where $b$ is a real non integer, yields

$$
f(x ; M)=\theta \lambda^{\theta} \sum_{i, j, k, \ell=0}^{\infty}(1+\ell) w_{i, j, k, \ell} x^{\theta-1} e^{-(\lambda x)^{\theta}(1+\ell)}
$$

Where,

$$
w_{i, j, k, \ell}=a b \alpha \beta(-1)^{i+j+k+\ell}\left(\begin{array}{c}
b-1 \\
i
\end{array}\right)\left(\begin{array}{c}
a+a i-1 \\
j
\end{array}\right)\left(\begin{array}{c}
\beta j+\beta-1 \\
k
\end{array}\right)\left(\begin{array}{c}
\alpha k+\alpha-1 \\
\ell
\end{array}\right)
$$

Condition for the expansion of the pdf of the Kw Kw W distribution Since,

$$
\int_{0}^{\infty} f(x ; M) d x=1
$$

Then,

$$
f(x ; M)=\theta \lambda^{\theta} \sum_{i, j, k, \ell=0}^{\infty}(1+\ell) w_{i, j, k, \ell} x^{\theta-1} e^{-(\lambda x)^{\theta}(1+\ell)}
$$

Where,

$$
w_{i, j, k, \ell}=a b \alpha \beta(-1)^{i+j+k+\ell}\left(\begin{array}{c}
b-1 \\
i
\end{array}\right)\left(\begin{array}{c}
a+a i-1 \\
j
\end{array}\right)\left(\begin{array}{c}
\beta j+\beta-1 \\
k
\end{array}\right)\left(\begin{array}{c}
\alpha k+\alpha-1 \\
\ell
\end{array}\right)
$$


and

$$
\sum_{i, j, k, \ell=0}^{\infty} w_{i, j, k, \ell}=1
$$

\subsection{An Expansion for The cdf}

We shall obtain an expansion for the cdf of the Kw Kw W distribution as follows:

Using binomial expansion in (5) yields

$$
F(x ; M)=1-\sum_{m, n, p, q=0}^{\infty}\left(\begin{array}{c}
b \\
m
\end{array}\right)\left(\begin{array}{c}
a m \\
n
\end{array}\right)\left(\begin{array}{c}
\beta n \\
p
\end{array}\right)\left(\begin{array}{c}
\alpha p \\
q
\end{array}\right)(-1)^{m+n+p+q} e^{-q(\lambda x)^{\theta}}
$$

Hence,

$$
F(x ; M)=1-\sum_{m, n, p, q=0}^{\infty} M_{m, n, p, q} e^{-q(\lambda x)^{\theta}}
$$

Where,

$$
M_{m, n, p, q}=\left(\begin{array}{c}
b \\
m
\end{array}\right)\left(\begin{array}{c}
a m \\
n
\end{array}\right)\left(\begin{array}{c}
\beta n \\
p
\end{array}\right)\left(\begin{array}{c}
\alpha p \\
q
\end{array}\right)(-1)^{m+n+p+q}
$$

\section{Some Properties of the Kw Kw W distribution}

In this section some properties of the $\mathrm{Kw} \mathrm{Kw} \mathrm{W}$ distribution will be obtained as follows:

\subsection{The $\mathbf{r}^{\text {th }}$ Moment}

Generally, the $\mathrm{r}^{\text {th }}$ moment of a random variable $\mathrm{X}$ can be given from

$$
E\left(X^{r}\right)=\int_{-\infty}^{\infty} x^{r} f(x ; M) d x
$$

Substituting (7) into the last equation yields

$$
E\left(X^{r}\right)=\theta \lambda^{\theta} \sum_{i, j, k, \ell=0}^{\infty}(1+\ell) w_{i, j, k, \ell} \int_{0}^{\infty} x^{r+\theta-1} e^{-(\lambda x)^{\theta}(1+\ell)} d x
$$

Hence,

$$
E\left(X^{r}\right)=\sum_{i, j, k, \ell=0}^{\infty} w_{i, j, k, \ell}\left(\frac{\lambda^{-\theta}}{1+\ell}\right)^{\frac{r}{\theta}} \Gamma\left(1+\frac{r}{\theta}\right)
$$

\subsection{The Moment Generating Function}

Generally, the moment generating function of a random variable $\mathrm{X}$ can be given from

$$
M_{x}(t)=\int_{-\infty}^{\infty} e^{t x} f(x ; M) d x
$$


Then,

$$
E\left(e^{t x}\right)=\sum_{r=0}^{\infty} \frac{t^{r}}{r !} \int_{-\infty}^{\infty} x^{r} f(x ; M) d x
$$

Hence,

$$
M_{x}(t)=\sum_{i, j, k, \ell=0}^{\infty} w_{i, j, k, \ell} \sum_{r=0}^{\infty}\left(\frac{t}{\lambda(1+\ell)^{1 / \theta}}\right)^{r} \frac{1}{r !} \Gamma\left(1+\frac{r}{\theta}\right)
$$

Using generalized hypergeometric function definition

$$
\left.{ }_{p} \psi_{q}\left[\begin{array}{c}
\left(\gamma_{1}, \mathrm{~A}_{1}\right), \ldots,\left(\gamma_{p}, \mathrm{~A}_{p}\right) \\
\left(\phi_{1}, \mathrm{~B}_{1}\right), \ldots,\left(\phi_{q}, \mathrm{~B}_{q}\right)
\end{array}\right] \mathrm{y}\right]=\sum_{n=0}^{\infty} \frac{\prod_{j=1}^{p} \Gamma\left(\gamma_{j}+A_{j} n\right) y^{n}}{\prod_{j=1}^{q} \Gamma\left(\phi_{j}+B_{j} n\right) n !}
$$

Then,

$$
\left.{ }_{1} \psi_{0}\left[\begin{array}{c}
\left(\gamma_{1}, \mathrm{~A}_{1}\right) \\
-
\end{array}\right] \mathrm{y}\right]=\sum_{n=0}^{\infty} \frac{y^{n} \Gamma\left(\gamma_{1}+A_{1} n\right)}{n !}
$$

Hence,

$$
M_{x}(t)=\sum_{i, j, k, \ell=0}^{\infty} w_{i, j, k, \ell} \psi_{0}\left[\begin{array}{c}
(1,1 / \theta) \\
-\frac{t}{\lambda(1+\ell)^{1 / \theta}}
\end{array}\right]
$$

\subsection{Quantile Function}

Starting with the well-known definition of the $100 \mathrm{q}^{\text {th }}$ quantile it is clear that

$$
q=P\left(X \leq x_{q}\right)=F\left(x_{q} ; \mathrm{M}\right) ; x_{q}>0,0<q<1
$$

Quantiles of the Kw Kw W distribution can be obtained by equating the cdf from (5) to q:

$$
q=1-\left\{1-\left[1-\left(1-\left(1-e^{-(\lambda x)^{\theta}}\right)^{\alpha}\right)^{\beta}\right]^{a}\right\}^{b}
$$

Then,

$$
x=\left\{-\lambda^{-\theta} \log \left\{1-\left[1-\left[1-\left(1-(1-q)^{\frac{1}{b}}\right)^{\frac{1}{a}}\right]^{\frac{1}{\beta}}\right\}^{\frac{1}{\alpha}}\right.\right.
$$

Easily, substituting q with $1 / 2$ (the second quantile) yields the median 


\subsection{The Mode}

The nature logarithm of (6) is

$$
\begin{aligned}
& I(x ; M)=\ln \left(a b \alpha \beta \theta \lambda^{\theta}\right)+(\theta-1) \ln (x)-[\lambda(x)]^{\theta}+(\alpha-1) \ln \left[1-e^{-[\lambda \cdot(x)]^{\theta}}\right] \\
&+(\beta-1) \ln \left[1-\left[1-e^{-[\lambda \cdot(x)]^{\theta}}\right]^{\alpha}\right]+(a-1) \ln \left[1-\left[1-\left[1-e^{-[\lambda(x)]^{\theta}}\right]^{\alpha}\right]^{\beta}\right] \\
&+(b-1)\left[1-\left[1-\left[1-\left[1-e^{-[\lambda(x)]^{\theta}}\right]^{\alpha}\right]^{\beta}\right]^{a}\right]
\end{aligned}
$$

Then, differentiating the nature logarithm of (6) with respect to $x$ and equating it to zero yields:

$$
\begin{aligned}
& \frac{\theta-1}{x}-\theta \lambda(\lambda x)^{\theta-1}-\frac{\theta \lambda e^{-[\lambda x]^{\theta}}(\alpha-1)(\lambda x)^{\theta-1}}{e^{-[\lambda . x]^{\theta}}-1}+\frac{\alpha \theta \lambda e^{-[\lambda x]^{\theta}}(\beta-1)\left[1-e^{-(\lambda x)^{\theta}}\right]^{\alpha-1}(\lambda x)^{\theta-1}}{\left[1-e^{-(\lambda x)^{\theta}}\right]^{\alpha}-1} \\
& -\frac{\beta \alpha \theta \lambda e^{-(\lambda x)^{\theta}}\left[1-\left[1-e^{-(\lambda x)^{\theta}}\right]^{\alpha}\right]^{\beta-1}(a-1)\left[1-e^{-(\lambda x)^{\theta}}\right]^{\alpha-1}(\lambda x)^{\theta-1}}{\left[1-e^{-(\lambda x)^{\theta}}\right]^{\alpha}-1} \\
& +\frac{a . \beta . \alpha \cdot \theta \cdot \lambda \cdot e^{-(\lambda . x)^{\theta}}\left[1-\left[1-e^{-(\lambda . x)^{\theta}}\right]^{\alpha}\right]^{\beta-1} \cdot(b-1) \cdot\left[1-e^{-(\lambda . x)^{\theta}}\right]^{\alpha-1}}{\left[1-\left[1-\left[1-e^{-(\lambda . x)^{\theta}}\right]^{\alpha}\right]^{\beta}\right]^{a}-1} \\
& \times(\lambda \cdot x)^{\theta-1}\left[1-\left[1-\left[1-e^{-(\lambda . x)^{\theta}}\right]^{\alpha}\right]^{\beta}\right]^{a-1}=0
\end{aligned}
$$

The last equation is a nonlinear equation and does not have an analytic solution with respect to $x$. Therefore, we have to solve it numerically. If $x_{0}$ is a root for the last equation it must be $\frac{\partial^{2}}{\partial x^{2}} \log \mathrm{f}\left(x_{0}\right)<0$

\subsection{The Mean Deviation}

Generally, the mean deviation about the mean and about the median for a random variable $\mathrm{X}$ respectively can be given from

$$
\delta_{1}(X)=\int_{-\infty}^{\infty}|x-\mu| f(x) d x \text { and } \delta_{2}(X)=\int_{-\infty}^{\infty}|x-\eta| f(x) d x
$$


Easily, we can obtain them respectively from

$$
\delta_{1}(X)=2 \mu F(\mu)-2 J(\mu) \text { and } \delta_{2}(X)=\mu-2 J(\eta)
$$

In order to obtain $\delta_{1}(X)$ and $\delta_{2}(X)$ we must obtain the following integration of $J($.$) ,$ where $J($.$) is called the incomplete first moment$

$$
J(Z)=\int_{0}^{z} x f(x ; M) d x
$$

Substituting (7) into the last equation yields

$$
J(x ; M)=\theta \lambda^{\theta} \sum_{i, j, k, \ell=0}^{\infty}(1+\ell) w_{i, j, k, \ell} \int_{0}^{z} x^{\theta} e^{-(\lambda x)^{\theta}(1+\ell)} d x
$$

Hence,

$$
J(z, M)=\sum_{i, j, k, \ell=0}^{\infty} \frac{w_{i, j, k, \ell}}{\lambda(1+\ell)^{\frac{1}{\theta}}} \Gamma\left(\frac{1}{\theta}+1,(1+\ell)(\lambda z)^{\theta}\right)
$$

\section{The Survival and Hazard Function}

Generally, the survival function of a random variable $\mathrm{X}$ can be given from

$$
S(x ; M)=1-F(x ; M)
$$

Substituting (5) into last equation yields

$$
S(x ; M)=\left\{1-\left[1-\left(1-\left(1-e^{-(\lambda x)^{\theta}}\right)^{\alpha}\right)^{\beta}\right]^{a}\right\}^{b}
$$

Generally, the Hazard function can be given from

$$
H(x ; M)=\frac{f(x ; M)}{S(x ; M)}
$$

so the hazard function of the $\mathrm{Kw} \mathrm{Kw}$ W distribution can be given by substituting (6) and (10) into last equation

$$
\frac{a b \alpha \beta \theta \lambda^{\theta} x^{\theta-1} e^{-(\lambda \theta)^{\theta}}\left(1-e^{-(\lambda x)^{\theta}}\right)^{\alpha-1}\left(1-\left(1-e^{-(\lambda x)^{\theta}}\right)^{\alpha}\right)^{\beta-1}\left[1-\left(1-\left(1-e^{-(\lambda x)^{\theta}}\right)^{\alpha}\right)^{\beta}\right]^{a-1}}{1-\left[1-\left(1-\left(1-e^{-(\lambda x)^{\theta}}\right)^{\alpha}\right)^{\beta}\right]^{a}}
$$




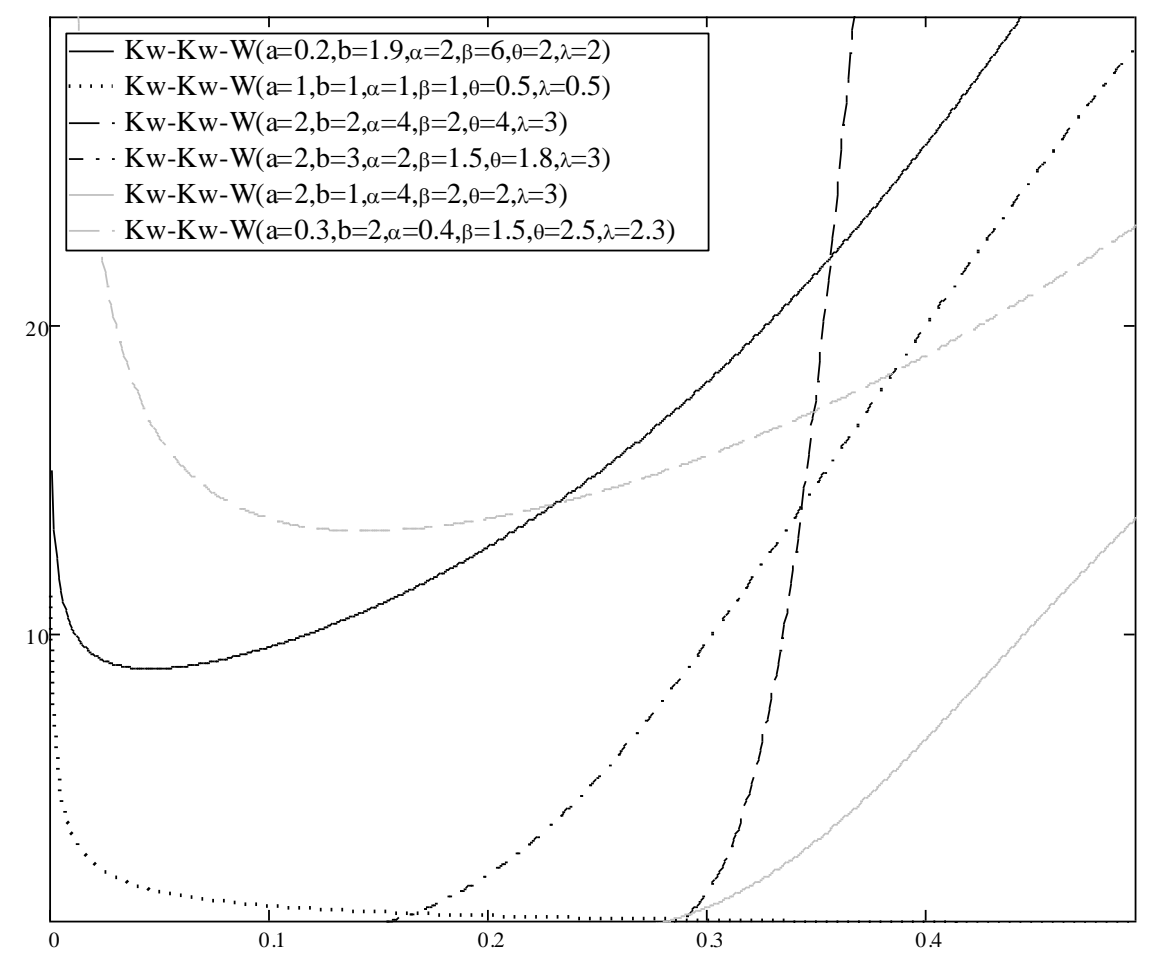

Figure 2: Plot of the $\mathrm{Kw} \mathrm{Kw} \mathrm{W}$ Hazard for some parameters values

We see from the last Figure that the Hazard rate function of the Kw Kw W distribution has many shapes like decreasing shapes, increasing shapes and decreasing then increasing shapes.

\section{Order Statistics}

A simple random sample $\mathrm{X}_{1}, \mathrm{X}_{2}, . ., \mathrm{X}_{\mathrm{v}}$ given from the $\mathrm{Kw} \mathrm{Kw} \mathrm{W}$ distribution where $\mathrm{X}^{\prime} \mathrm{s}$ are i.i.d random variables, has the density $\mathrm{f}_{\mathrm{u}: \mathrm{v}}\left(x_{\mathrm{u}: \mathrm{v}}\right)$ of the $\mathrm{u}^{\text {th }}$ order statistic, for $\mathrm{u}=1,2, \ldots \ldots, \mathrm{v}$

as follows, see Arnold, et al.(1992)

$$
f_{u, v}\left(x_{u, v}\right)=\frac{f\left(x_{u}\right)}{B(u, v-u+1)} F\left(x_{u}\right)^{u-1}\left\{1-F\left(x_{u}\right)\right\}^{v-u}
$$

Since,

$$
\left[1-F\left(x_{u}\right)\right]^{v-u}\left[F\left(x_{u}\right)\right]^{u-1}=\sum_{m=0}^{u-1} \sum_{n, p, q, s=0}^{\infty} N_{m, n, p, q, s} e^{-s\left(\lambda x_{u}\right)^{\theta}}
$$

Where,

$$
N_{m, n, p, q, s}=\left(\begin{array}{c}
u-1 \\
m
\end{array}\right)\left(\begin{array}{c}
b(m+v-u) \\
n
\end{array}\right)\left(\begin{array}{c}
a n \\
p
\end{array}\right)\left(\begin{array}{c}
\beta p \\
q
\end{array}\right)\left(\begin{array}{c}
\alpha q \\
s
\end{array}\right)(-1)^{m+n+p+q+s}
$$


Substituting (15) and (7) into (14) yields the density $f_{u: v}\left(x_{u: v}\right)$ of the $\mathrm{u}^{\text {th }}$ order statistic

$$
\begin{aligned}
f_{u v}\left(x_{u v} ; M\right) & =\frac{1}{B(u, v-u+1)} \theta \lambda^{\theta} \sum_{i, j, k, l=0}^{\infty} \sum_{m=0}^{u-1} \sum_{n, p, q, s=0}^{\infty}(1+\ell) w_{i, j, k, \ell} \\
& \times N_{m, n, p, q, s} x_{u}^{\theta-1} e^{-\left(\lambda x_{u}\right)^{\theta}(1+\ell+s)}
\end{aligned}
$$

Moments of order statistics

Generally, the moments of order statistics can be given from

Substituting (16) into last equation gives

$$
\begin{aligned}
E_{u, v}\left(X_{u v}^{r}\right)= & \frac{1}{B(u, v-u+1)} \theta \lambda^{\theta} \sum_{i, j, k, l=0}^{\infty} \sum_{m=0}^{u-1} \sum_{n, p, q, s=0}^{\infty}(1+\ell) w_{i, j, k, \ell} N_{m, n, p, q, s} \\
& \times \int_{0}^{\infty} x_{u}^{r+\theta-1} e^{-\left(\lambda x_{u}\right)^{\theta}(1+\ell+s)} d x_{u}
\end{aligned}
$$

Let $I=\int_{0}^{\infty} x_{u}^{r+\theta-1} e^{-\left(\lambda x_{u}\right)^{\theta}(1+\ell+s)} d x_{u}$

Then,

$$
I=\frac{\lambda^{-r-\theta}}{\theta(1+\ell+s)^{1+\frac{r}{\theta}}} \Gamma\left(1+\frac{r}{\theta}\right)
$$

Substituting (18) into (17) yields

$$
E_{u, v}\left(X_{u, v}^{r}\right)=\frac{1}{B(u, v-u+1)} \sum_{i, j, k, l=0}^{\infty} \sum_{m=0}^{u-1} \sum_{n, p, q, s=0}^{\infty}(1+\ell) w_{i, j, k, \ell} N_{m, n, p, q, s} \frac{\lambda^{-r}}{(1+\ell+s)^{1+\frac{r}{\theta}}} \Gamma\left(1+\frac{r}{\theta}\right)
$$

L-moments are linear functions of expected order statistics where L-moments is defined as, see Hosking (1990):

$$
\lambda_{\alpha+1}=(\alpha+1)^{-1} \sum_{t=0}^{\alpha}(-1)^{t}\left(\begin{array}{l}
\alpha \\
t
\end{array}\right) E\left(x_{\alpha+1-t: \alpha+1}\right), \alpha=0,1, \ldots
$$

the first L-moments is

$$
\lambda_{1}=E\left(X_{1: 1}\right)
$$

Where,

$$
E_{1: 1}\left(X_{1: 1}^{r}\right)=\sum_{i, j, k, l=0}^{\infty} \sum_{n, p, q, s=0}^{\infty}(1+\ell) w_{i, j, k, \ell} N_{n, p, q, s} \frac{\lambda^{-r}}{(1+\ell+s)^{1+\frac{r}{\theta}}} \Gamma\left(1+\frac{r}{\theta}\right)
$$


Similarly $\lambda_{2}, \lambda_{3}$ and $\lambda_{4}$ can be obtained.

$$
\begin{aligned}
& \lambda_{2}=\frac{1}{2} E\left(X_{2: 2}-2 X_{1: 2}\right) \\
& \lambda_{3}=\frac{1}{3} E\left(X_{3: 3}-2 X_{2: 3}+X_{1: 3}\right) \\
& \lambda_{4}=\frac{1}{4} E\left(X_{4: 4}-3 X_{3: 4}+3 X_{2: 4}-X_{1: 4}\right)
\end{aligned}
$$

\section{The Asymptotes of $F(X)$ and $f(X)$}

In this section, we shall obtain the asymptotes of the cdf and pdf of the $\mathrm{Kw} \mathrm{Kw} \mathrm{W}$ distribution

\subsection{The Asymptotes of cdf}

First: when $\mathrm{x}$ converges to zero

Using Maclaurin series expansion of the cdf gives

$$
\lim _{x \rightarrow 0} F(x ; M) \approx 1-\left\{1-\left(1-\left(1-(\lambda x)^{\theta \alpha}\right)^{\beta}\right)^{a}\right\}^{b}
$$

and using binomial expansion gives

$$
\lim _{x \rightarrow 0} F(x ; M) \approx b \beta^{a}(\lambda x)^{\theta \alpha a}
$$

Second: when $\mathrm{x}$ converges to $\infty$

Using binomial expansion of the cdf gives

Then,

$$
\lim _{x \rightarrow \infty} F(x ; M) \approx 1-\left[a \alpha^{\beta} e^{-\beta(\lambda x)^{\theta}}\right]^{b}
$$

Hence,

$$
1-\lim _{x \rightarrow \infty} F(x ; M) \approx a^{b} \alpha^{b \beta} e^{-b \beta(\lambda x)^{\theta}}
$$

\subsection{The Asymptotes of the pdf}

First: when x converges to zero.

$$
\lim _{x \rightarrow 0} f(x ; M) \approx a b \alpha \beta \theta \lambda^{\theta} x^{\theta-1}\left(1-e^{-(\lambda x)^{\theta}}\right)^{\alpha-1}\left[1-\left(1-\left(1-e^{-(\lambda x)^{\theta}}\right)^{\alpha}\right)^{\beta}\right]^{a-1}
$$

Using Maclaurin series expansion of the cdf gives

$$
\lim _{x \rightarrow 0} f(x ; M)=a b \alpha \beta \theta \lambda^{\theta} x^{\theta-1}(\lambda x)^{\theta(\alpha-1)}\left(1-\left(1-(\lambda x)^{\alpha \theta}\right)^{\beta}\right)^{\alpha-1}
$$


and using only first and second terms of cdf binomial expansion gives

$$
\lim _{x \rightarrow 0} f(x ; M) \approx a b \alpha \beta^{a} \theta \lambda^{\alpha a \theta} x^{\alpha a \theta-1}
$$

Second: when $\mathrm{x}$ converges to $\infty$

$\lim _{x \rightarrow \infty} f(x ; M) \approx a b \alpha \beta \theta \lambda^{\theta} x^{\theta-1} e^{-(\lambda x)^{\theta}}\left(1-\left(1-e^{-(\lambda x)^{\theta}}\right)^{\alpha}\right)^{\beta-1}\left\{1-\left[1-\left(1-\left(1-e^{-(\lambda x)^{\theta}}\right)^{\alpha}\right)^{\beta}\right]^{a}\right\}^{b-1}$

Using binomial expansion gives

$$
\lim _{x \rightarrow \infty} f(x ; M) \approx a^{b} b \alpha^{\beta b-1} \beta \theta \lambda^{\theta} x^{\theta-1} e^{-(\lambda x)^{\theta}(\beta+b-1)}
$$

\section{Extreme Values}

If $X_{1}, X_{2}, \ldots, X_{n}$ is a random sample from (6) and if $\bar{X}=\left(X_{1}+X_{2}+\ldots+X_{n}\right) / n$ denotes the sample mean, then according to central limit theorem the quantity $\sqrt{n}(\bar{X}-E(X)) / \sqrt{\operatorname{Var}(X)}$ approaches the standard normal distribution where $n \rightarrow \infty$ In this section, we will obtain some properties of the asymptotes of the extreme values $M_{n}=\max \left(X_{1}, X_{2}, \ldots, X_{n}\right)$ and $m_{n}=\min \left(X_{1}, X_{2}, \ldots, X_{n}\right)$.

First Minimum value:

It is known from (19) that

$$
\lim _{x \rightarrow 0} \frac{F(t x)}{F(t)}=\frac{(\lambda t)^{\theta \alpha a} x^{\theta \alpha a}}{(\lambda t)^{\theta \alpha a}}=x^{\theta \alpha a}
$$

It follows from theorem1.6.2 in Leadbetter et al.(1987) that there must be norming constants $\gamma_{n}$ and $\delta_{n}$, such that

$$
p r\left\{\gamma_{n}\left(m_{n}-\delta_{n}\right) x\right\} \rightarrow e^{-x^{\theta \alpha a}} \text { As } n \rightarrow \infty
$$

The form of the norming constants $\gamma_{n}$ and $\delta_{n}$ can be determined. For instance, using corollary 1.6.3 in Leadbetter et al. (1987), gives

$$
\gamma_{n}=F^{-1}(0)=0 \text { and } \delta_{n}=F^{-1}\left(\frac{1}{n}\right)-F^{-1}(0)=\frac{1}{\lambda}\left[\frac{1}{n b \beta^{a}}\right]^{\frac{1}{\theta \alpha a}}
$$

Second Maximum value:

Since,

$$
\lim _{t \rightarrow \infty} \frac{1-F(t+x g(t))}{1-F(t)}=\lim _{t \rightarrow \infty} \frac{e^{-b \beta \lambda^{\theta} t^{\theta}\left(1+\frac{x g(t)}{t}\right)^{\theta}}}{e^{-b \beta(\lambda t)^{\theta}}}
$$


and using the binomial expansion yields

$$
\lim _{t \rightarrow \infty} \frac{1-F(t+x g(t))}{1-F(t)}=\lim _{t \rightarrow \infty} \frac{e^{-b \beta \lambda^{\theta} t^{\theta}\left(1+\theta \frac{x g(t)}{t}\right)}}{e^{-b \beta(\lambda t)^{\theta}}}=\lim _{t \rightarrow \infty} e^{\frac{-b \beta \lambda^{\theta} t^{\theta} \theta x g(t)}{t}}
$$

Then,

$$
\lim _{t \rightarrow \infty} \frac{1-F(t+x g(t))}{1-F(t)}=e^{-x} \text { Where, } g(t)=\left[\frac{b \beta \lambda^{\theta} t^{\theta} \theta}{t}\right]^{-1}
$$

it follows from theorem1.6.2 in Leadbetter et al.(1987) that there must be norming constants $\psi_{n}$ and $\omega_{n}$, such that

$$
\operatorname{pr}\left\{\psi_{n}\left(M_{n}-\omega_{n}\right) \leq x\right\} \rightarrow e^{-e^{-x}} \text { As } n \rightarrow \infty
$$

The form of the norming constants $\psi_{n}$ and $\omega_{n}$ can be determined. Using Corollary 1.6.3 in Leadbetter et al. (1987) yields that

$$
\begin{aligned}
& \psi_{n}=\left[g\left(\omega_{n}\right)\right]^{-1}=\frac{\theta b \beta \lambda^{\theta} d_{n}^{\theta}}{d_{n}}=\theta b \beta \lambda^{\theta} d_{n}^{\theta-1} \text { and } \\
& \omega_{n}=F^{-1}\left(1-\frac{1}{n}\right)=\frac{1}{\lambda}\left[\frac{-1}{b \beta} \ln \frac{\left(1-\left(1-\frac{1}{n}\right)\right)}{a^{b} \alpha^{\beta b}}\right]^{\frac{1}{\theta}}
\end{aligned}
$$

Hence,

$$
\omega_{n}=\frac{1}{\lambda(b \beta)^{\frac{1}{\theta}}}\left[\ln \mathrm{n}+\ln a^{b} \alpha^{\beta b}\right]^{\frac{1}{\theta}}
$$

\section{The Maximum Likelihood Estimation}

We now determine the maximum likelihood estimates (MLEs) of the parameters of the $\mathrm{Kw} \mathrm{Kw} \mathrm{W}$ distribution from complete samples. Let $x_{1}, x_{2}, \ldots, x_{\mathrm{n}}$ be a random sample of size $\mathrm{n}$ from the $\mathrm{Kw} \mathrm{Kw} \mathrm{W}(\mathrm{x} ; M)$ distribution, where $M=(a, b, \alpha, \beta, \theta, \lambda)$

The log-likelihood function for the vector of parameters $(a, b, \alpha, \beta, \theta$ and $\lambda)$ can be written as

$$
\begin{aligned}
& \ell(M ; x)=n \log \left[a b \alpha \beta \theta \lambda^{\theta}\right]+(\theta-1) \sum_{i=1}^{n} \log x_{i}-\sum_{i=1}^{n}\left(\lambda x_{i}\right)^{\theta}+(\alpha-1) \sum_{i=1}^{n} \log \left(1-e^{-\left(\lambda x_{i}\right)^{\theta}}\right) \\
& \quad+(\beta-1) \sum_{i=1}^{n} \log \left[1-\left(1-e^{-\left(\lambda x_{i}\right)^{\theta}}\right)^{\alpha}\right]+(a-1) \sum_{i=1}^{n} \log \left[1-\left(1-\left(1-e^{-\left(\lambda x_{i}\right)^{\theta}}\right)^{\alpha}\right)^{\beta}\right] \\
& \quad+(b-1) \sum_{i=1}^{n} \log \left[1-\left(1-\left(1-\left(1-e^{-\left(\lambda x_{i}\right)^{\theta}}\right)^{\alpha}\right)^{\beta}\right)^{a}\right]
\end{aligned}
$$


The score functions for the parameters $a, b, \alpha, \beta, \theta$ and $\lambda$ are given by

$$
\begin{aligned}
\frac{\partial}{\partial a} \ell(M ; x) & =\frac{n}{a}+\sum_{i=1}^{n} \log \left[1-\left[1-\left[1-e^{-\left[\lambda x_{i}\right]^{\theta}}\right]^{\alpha}\right]^{\beta}\right] \\
+ & \sum_{i=1}^{n} \frac{(b-1) \log \left[1-\left[1-\left[1-e^{-\left[\lambda x_{i}\right]^{\theta}}\right]^{\alpha}\right]^{\beta}\right]\left[1-\left[1-\left[1-e^{-\left[\lambda x_{i}\right]^{\theta}}\right]^{\alpha}\right]^{\beta}\right]^{a}}{\left[1-\left[1-\left[1-e^{-\left[\lambda x_{i}\right]^{\theta}}\right]^{\alpha}\right]^{\beta}\right]^{a}-1}, \\
\frac{\partial}{\partial b} \ell(M ; x) & =\frac{n}{b}+\sum_{i=1}^{n} \log \left[1-\left[1-\left[1-\left[1-e^{-\left[\lambda x_{i}\right]^{\theta}}\right]^{\alpha}\right]^{\beta}\right]^{a}\right]
\end{aligned}
$$

$$
\frac{\partial}{\partial \beta} \ell(M ; x)=\sum_{i=1}^{n} \log \left[1-\left[1-e^{-\left[\lambda x_{i}\right]^{\theta}}\right]^{\alpha}\right]+\frac{n}{\beta}+(a-1) \sum_{i=1}^{n} \frac{\left[1-\left[1-e^{-\left[\lambda x_{i}\right]^{\theta}}\right]^{\alpha}\right]^{\beta} \log \left[1-\left[1-e^{-\left[\lambda x_{i}\right]^{\theta}}\right]^{\alpha}\right]}{\left[1-\left[1-e^{-\left[\lambda x_{i}\right]^{\theta}}\right]^{\alpha}\right]^{\beta}-1}
$$

$$
-a(b-1) \sum_{i=1}^{n} \frac{\left[1-\left[1-e^{-\left[\lambda x_{i}\right]^{\theta}}\right]^{\alpha}\right]^{\beta}\left[1-\left[1-\left[1-e^{-\left[\lambda x_{i}\right]^{\theta}}\right]^{\alpha}\right]^{\beta}\right]^{a-1} \log \left[1-\left[1-e^{-\left[\lambda x_{i}\right]^{\theta}}\right]^{\alpha}\right]}{\left[1-\left[1-\left[1-e^{-\left[\lambda x_{i}\right]^{\theta}}\right]^{\alpha}\right]^{\beta}\right]^{a}-1},
$$$$
\frac{\partial}{\partial \alpha} \ell(M ; x)=\frac{n}{\alpha}+\sum_{i=1}^{n} \log \left[1-e^{-\left[\lambda x_{i}\right]^{\theta}}\right]+(\beta-1) \sum_{i=1}^{n} \frac{\left[1-e^{-\left[\lambda x_{i}\right]^{\theta}}\right]^{\alpha} \log \left[1-e^{-\left[\lambda x_{i}\right]^{\theta}}\right]}{\left[1-e^{-[\lambda x]^{\theta}}\right]^{\alpha}-1}
$$

$$
\begin{aligned}
& -\beta(a-1) \sum_{i=1}^{n} \frac{\left[1-\left[1-e^{-\left[\lambda x_{i}\right]^{\theta}}\right]^{\alpha}\right]^{\beta-1}\left[1-e^{-\left[\lambda x_{i}\right]^{\theta}}\right]^{\alpha} \log \left[1-e^{-\left[\lambda x_{i}\right]^{\theta}}\right]}{\left[1-\left[1-e^{-\left[\lambda x_{i}\right]^{\theta}}\right]^{\alpha}\right]^{\beta}-1} \\
& +a \beta(b-1) \sum_{i=1}^{n} \frac{\left[1-\left[1-e^{-\left[\lambda x_{i}\right]^{\theta}}\right]^{\alpha}\right]^{\beta-1}\left[1-e^{-\left[\lambda x_{i}\right]^{\theta}}\right]^{\alpha} \log \left[1-e^{-\left[\lambda x_{i}\right]^{\theta}}\right]}{\left.\left[1-\left[1-e^{-\left[\lambda x_{i}\right]^{\theta}}\right]^{\alpha}\right]^{\beta}\right]^{a}-1} \\
& \times\left[1-\left[1-\left[1-e^{-\left[\lambda x_{i}\right]^{\theta}}\right]^{\alpha}\right]^{\beta}\right]^{a-1},
\end{aligned}
$$




$$
\begin{aligned}
\frac{\partial}{\partial \lambda} \ell(M ; x) & =\frac{\theta \lambda^{\theta-1} n}{\lambda^{\theta}}-\theta \lambda^{\theta-1} \sum_{i=1}^{n} x_{i}^{\theta}-\theta(\alpha-1) \lambda^{\theta-1} \sum_{i=1}^{n} \frac{x_{i} e^{\theta} e^{-\left[\lambda x_{i}\right]^{\theta}}-1}{\left.x_{i}\right]^{\theta}} \\
& +\lambda^{\theta-1} \alpha \theta(\beta-1) \sum_{i=1}^{n} \frac{x^{\theta} e^{-\left[\lambda x_{i}\right]^{\theta}}\left[1-e^{-\left[\lambda x_{i}\right]^{\theta}}\right]^{\alpha-1}}{\left[1-e^{-\left[\lambda x_{i}\right]^{\theta}}\right]^{\alpha}-1} \\
& -\beta \alpha \theta(a-1) \lambda^{\theta-1} \sum_{i=1}^{n} \frac{x_{i}^{\theta} e^{-\left[\lambda x_{i}\right]^{\theta}}\left[1-\left[1-e^{-\left[\lambda x_{i}\right]^{\theta}}\right]^{\alpha}\right]^{\beta-1}\left[1-e^{-\left[\lambda x_{i}\right]^{\theta}}\right]^{\alpha-1}}{\left[1-\left[1-e^{-\left[\lambda x_{i}\right]^{\theta}}\right]^{\alpha}\right]^{\beta}-1} \\
& +a \beta \alpha \theta(b-1) \lambda^{\theta-1} \sum_{i=1}^{n} \frac{x_{i}^{\theta} e^{-\left[\lambda x_{i}\right]^{\theta}}\left[1-\left[1-e^{-\left[\lambda x_{i}\right]^{\theta}}\right]^{\alpha}\right]^{\beta-1}\left[1-e^{-\left[\lambda x_{i}\right]^{\theta}}\right]^{\alpha-1}\left[1-\left[1-\left[1-e^{-\left[\lambda x_{i}\right]^{\theta}}\right]^{\alpha}\right]^{\beta}\right]^{a}-1}{\left.\left.-\left[1-e^{-\left[\lambda x_{i}\right]^{\theta}}\right]^{\alpha}\right]^{\beta}\right]^{a-1}}
\end{aligned}
$$

and

$$
\begin{aligned}
\frac{\partial}{\partial \theta} \ell(M ; x) & =\sum_{i=1}^{n} \log \left(x_{i}\right)-\lambda^{\theta} \sum_{i=1}^{n} x_{i}{ }^{\theta} \log \left[\lambda x_{i}\right]-(\alpha-1) \lambda^{\theta} \sum_{i=1}^{n} \frac{e^{-\left[\lambda x_{i}\right]^{\theta}} x_{i}{ }^{\theta} \log \left[\lambda x_{i}\right]}{e^{-\left[\lambda x_{i}\right]^{\theta}}-1} \\
& +\alpha(\beta-1) \lambda^{\theta} \sum_{i=1}^{n} \frac{e^{-\left[\lambda x_{i}\right]^{\theta}}\left[1-e^{-\left[\lambda x_{i}\right]^{\theta}}\right]^{\alpha-1} x_{i}^{\theta} \log \left[\lambda x_{i}\right]}{\left[1-e^{-\left[\lambda x_{i}\right]^{\theta}}\right]^{\alpha}-1}+n \sum_{i=1}^{n} \frac{1+\theta \log \lambda}{\theta} \\
& -\beta \alpha(a-1) \lambda^{\theta} \sum_{i=1}^{n} \frac{x^{\theta} e^{-\left[\lambda x_{i}\right]^{\theta}}\left[1-\left[1-e^{-\left[\lambda x_{i}\right]^{\theta}}\right]^{\alpha}\right]^{\beta-1}\left[1-e^{-\left[\lambda x_{i}\right]^{\theta}}\right]^{\alpha-1} \log \left[\lambda x_{i}\right]}{\left[1-\left[1-e^{-\left[\lambda x_{i}\right]^{\theta}}\right]^{\alpha}\right]^{\beta}-1} \\
& +a \beta \alpha(b-1) \lambda^{\theta} \sum_{i=1}^{n} \frac{x^{\theta} e^{-\left[\lambda x_{i}\right]^{\theta}}\left[1-\left[1-e^{-\left[\lambda x_{i}\right]^{\theta}}\right]^{\alpha}\right]^{\beta-1}\left[1-e^{-\left[\lambda x_{i}\right]^{\theta}}\right]^{\alpha-1}\left[1-\left[1-\left[1-e^{-\left[\lambda x_{i}\right]^{\theta}}\right]^{\alpha}\right]^{\beta}\right]^{a-1} \log \left[\lambda x_{i}\right]}{\left.\left[1-\left[1-e^{-\left[\lambda x_{i}\right]^{\theta}}\right]^{\alpha}\right]^{\beta}\right]^{a}-1}
\end{aligned}
$$

The MLEs of the unknown parameters are obtained by solving the nonlinear likelihood equations from (21) to (26) but They cannot be solved analytically, so we shall use a statistical software to solve the equations numerically. We can use iterative techniques such as a Newton-Raphson algorithm to obtain the estimate. 


\section{The Variance-Covariance Matrix}

Let $\theta$ is the vector of the unknown parameters $(a, b, \alpha, \beta, \theta, \lambda)$, The element of the $6 \times 6$ information matrix $I(a, b, \alpha, \beta, \theta, \lambda)$ can be approximated by:

$$
I_{i j}(\hat{\theta})=-\left.\frac{\partial^{2} \ell(\theta)}{\partial \theta_{i} \partial \theta_{j}}\right|_{\theta=\hat{\theta}}
$$

and $I^{1}(a, b, \alpha, \beta, \theta, \lambda)$ is the variance-covariance matrix of the unknown parameters, where second derivatives can be easily driven via Mathcad, Maple, Matlab, or R.

The asymptotic distributions of the MLE parameters

$$
\sqrt{n}\left(\hat{\theta}_{i}-\theta_{i}\right) \approx N_{6}\left(0, I^{-1}\left(\hat{\theta}_{i}\right)\right), i=1, \ldots ., 6
$$

The approximation $100(1-\gamma) \%$ confidence intervals of the unknown parameters based on the asymptotic distribution of the $\mathrm{Kw} \mathrm{Kw} \mathrm{W}(a, b, \alpha, \beta, \theta, \lambda)$ distribution are determined respectively as

$$
\hat{\theta}_{i} \pm Z_{\frac{\gamma}{2}} \sqrt{I^{-1}\left(\hat{\theta}_{i}\right)}, i=1, \ldots ., 6
$$

\section{Illustration Study}

An experiment is given to illustrate new results of the $\mathrm{Kw} \mathrm{Kw} W(a, b, \alpha, \beta, \theta, \lambda)$ distribution, This study is about MLEs of parameters of the $\mathrm{Kw} \mathrm{Kw} W$ distribution. The algorithm of obtaining parameters estimates is described in the following steps:

Step (1): Generate a random sample of size $n$ as follows: $\mathrm{u}_{1}, \mathrm{u}_{2}, \ldots, \mathrm{u}_{\mathrm{n}}$, by using the uniform distribution $(0,1)$

Step (2): transform the uniform random numbers to random numbers of the $\mathrm{Kw} \mathrm{Kw}$ $\mathrm{W}$ distribution by using the quantile function of the $\mathrm{Kw} \mathrm{Kw} \mathrm{W}$ distribution:

Step (3): Solve the 21 to 26 by iteration to get the maximum likelihood estimators via iterative techniques and repeat it many times.

In this experiment $10,15,30$ and 50 random numbers were generated using Mathcad package, then we obtained MLEs the $\mathrm{Kw} \mathrm{Kw} \mathrm{W}(a, b, \alpha, \beta, \theta, \lambda)$ distribution, where we started with parameters values: $a=2, b=5, \alpha=4, \beta=3, \theta=2$ and $\lambda=3$ for 1000 times, then we used the conjugate gradient iteration method. Finally, we get the following results 


\begin{tabular}{|c|c|c|c|}
\hline Sample size & Parameters & Biases & RMSES \\
\hline \multirow{6}{*}{10} & $a$ & 0.226 & 1.668 \\
\hline & $b$ & -1.452 & 6.02 \\
\hline & $\alpha$ & 1.449 & 2.291 \\
\hline & $\beta$ & 1.038 & 2.228 \\
\hline & $\theta$ & 2.418 & 3.422 \\
\hline & $\lambda$ & 0.425 & 1.176 \\
\hline \multirow{6}{*}{15} & $a$ & 0.132 & 1.57 \\
\hline & $b$ & -0.986 & 3.571 \\
\hline & $\alpha$ & 1.268 & 2.14 \\
\hline & $\beta$ & 0.98 & 2.102 \\
\hline & $\theta$ & 2.246 & 3.236 \\
\hline & $\lambda$ & 0.342 & 1.017 \\
\hline \multirow{6}{*}{30} & $a$ & -0.074 & 1.269 \\
\hline & $b$ & -0.49 & 3.204 \\
\hline & $\alpha$ & 0.788 & 1.57 \\
\hline & $\beta$ & 0.572 & 1.621 \\
\hline & $\theta$ & 1.413 & 2.345 \\
\hline & $\lambda$ & 0.257 & 0.994 \\
\hline \multirow{6}{*}{50} & $a$ & -0.131 & 1.07 \\
\hline & $b$ & -0.107 & 2.64 \\
\hline & $\alpha$ & 0.533 & 1.339 \\
\hline & $\beta$ & 0.411 & 1.316 \\
\hline & $\theta$ & 0.97 & 1.762 \\
\hline & $\lambda$ & 0.164 & 0.871 \\
\hline
\end{tabular}

We see that the more sample size increases the more Biases and RMSEs decrease.

\section{Application}

In this section we give a real data to illustrate an example for one distribution of the new family of $\mathrm{Kw} \mathrm{Kw}$ distributions so called $\mathrm{Kw} \mathrm{Kw} \mathrm{W}$ distribution to see how the new model works practically and we shall use the Mathcad package version 15 to do that. In our example, we used different distributions as the kumaraswamy - kumaraswamy Weibull ( $\mathrm{Kw} \mathrm{Kw} \mathrm{W}$ ) distribution [derived from the $\mathrm{Kw} \mathrm{Kw}$ family], the exponentiated kumaraswamy Weibull ( $\mathrm{E} \mathrm{KW} \mathrm{W}$ ) distribution [derived from the $\mathrm{E} \mathrm{KW}$ family], the kumaraswamy Weibull ( $\mathrm{Kw} \mathrm{W}$ ) distribution [derived from the $\mathrm{Kw}$ family], the exponentiated generalized Weibull (EG W) distribution [derived from the E G family], the exponentiated Weibull (E-W) distribution [derived from the $\mathrm{E}$ family] and the 
Weibull (W) distribution. The following data represents the lifetime (Hours) of T8 fluorescent lamps for 30 devices

$0.228 \times 10^{5}, 0.25 \times 10^{5}, 0.256 \times 10^{5}, 0.262 \times 10^{5}, 0.263 \times 10^{5}, 0.263 \times 10^{5}, 0.264 \times 10^{5}$, $0.267 \times 10^{5}, 0.272 \times 10^{5}, 0.274 \times 10^{5}, 0.277 \times 10^{5}, 0.288 \times 10^{5}, 0.288 \times 10^{5}, 0.295 \times 10^{5}$, $0.297 \times 10^{5}, 0.298 \times 10^{5}, 0.302 \times 10^{5}, 0.304 \times 10^{5}, 0.305 \times 10^{5}, 0.305 \times 10^{5}, 0.31 \times 10^{5}, 0.31 \times 10^{5}$, $0.311 \times 10^{5}, 0.315 \times 10^{5}, 0.325 \times 10^{5}, 0.329 \times 10^{5}, 0.349 \times 10^{5}, 0.352 \times 10^{5}, 0.359 \times 10^{5}$, $0.384 \times 10^{5}$

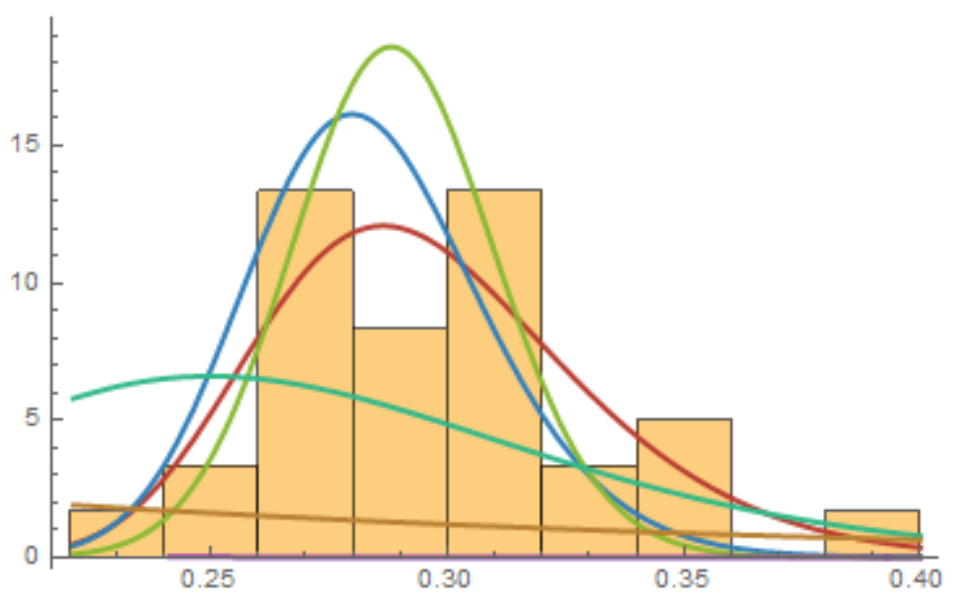

- KW-KW-Weibull

- E-KW-Weibull

- KW-Weibull

- EG-Weibull

- E-Weibull

- Weibull

Figure 3. Probability density functions for different distributions

Table 1: The MLE of the parameter(s) and the associated AIC and BIC values

\begin{tabular}{|c|c|c|c|c|c|c|c|c|c|c|c|}
\hline \multirow{2}{*}{ The Model } & \multicolumn{6}{|c|}{ MLE of parameters } & \multirow{2}{*}{ K.S } & \multirow{2}{*}{$\mathrm{p}$-value } & \multirow{2}{*}{ AIC } & \multirow{2}{*}{ CAIC } & \multirow{2}{*}{ BIC } \\
\hline & A & b & $\alpha$ & $\beta$ & $\theta$ & $\Lambda$ & & & & & \\
\hline $\mathrm{Kw}$ Kw W (a, b, $\alpha, \beta, \theta, \lambda)$ & $\begin{array}{c}4.998 \\
(1.752)\end{array}$ & $\begin{array}{c}0.482 \\
(1.6)\end{array}$ & $\begin{array}{c}5.116 \\
(0.227)\end{array}$ & $\begin{array}{c}4.55 \\
(0.132)\end{array}$ & $\begin{array}{c}2.431 \\
(0.762)\end{array}$ & $\begin{array}{c}4.537 \\
(0.714)\end{array}$ & 0.087 & 0.023 & -86.104 & -82.452 & -77.697 \\
\hline $\mathrm{E} \mathrm{Kw} W \quad(a, \alpha, \beta, \theta, \lambda)$ & $\begin{array}{c}5.01 \\
(1.724)\end{array}$ & $\begin{array}{l}1 \\
-\end{array}$ & $\begin{array}{c}5.366 \\
(1.146)\end{array}$ & $\begin{array}{c}4.296 \\
(0.426)\end{array}$ & $\begin{array}{c}2.432 \\
(0.418)\end{array}$ & $\begin{array}{c}4.506 \\
(1.007)\end{array}$ & 0.254 & 0.958 & -73.684 & -71.184 & -66.678 \\
\hline $\mathrm{Kw} \mathrm{W}(\alpha, \beta, \theta, \lambda)$ & $\begin{array}{l}1 \\
-\end{array}$ & $\begin{array}{l}1 \\
-\end{array}$ & $\begin{array}{c}3.792 \\
(3.285)\end{array}$ & $\begin{array}{c}3.735 \\
(1.864)\end{array}$ & $\begin{array}{c}2.602 \\
(1.479)\end{array}$ & $\begin{array}{c}5.265 \\
(1.129)\end{array}$ & 0.931 & 0.998 & -59.802 & -58.202 & -54.197 \\
\hline EG W (a, $\beta, \theta, \lambda)$ & $\begin{array}{c}7.543 \\
(3.355)\end{array}$ & $\begin{array}{l}1 \\
\text { () }\end{array}$ & $\begin{array}{l}1 \\
\text { () }\end{array}$ & $\begin{array}{c}6.418 \\
(0.747)\end{array}$ & $\begin{array}{c}1.851 \\
(1.671)\end{array}$ & $\begin{array}{c}5.592 \\
(0.222)\end{array}$ & 0.999 & 0.999 & -69.588 & -67.988 & -63.983 \\
\hline $\mathrm{E} \quad \mathrm{W}(\alpha, \theta, \lambda)$ & $\begin{array}{l}1 \\
-\end{array}$ & $\begin{array}{l}1 \\
-\end{array}$ & $\begin{array}{c}7.201 \\
(4.117)\end{array}$ & $\begin{array}{l}1 \\
-\end{array}$ & $\begin{array}{c}1.949 \\
(2.107)\end{array}$ & $\begin{array}{c}5.987 \\
(1.421)\end{array}$ & 0.406 & 0.999 & -25.39 & -24.467 & -21.186 \\
\hline $\mathrm{W}(\theta, \lambda)$ & $\begin{array}{l}1 \\
-\end{array}$ & $\begin{array}{l}1 \\
-\end{array}$ & $\begin{array}{l}1 \\
-\end{array}$ & $\begin{array}{l}1 \\
-\end{array}$ & $\begin{array}{c}1.163 \\
(2.473)\end{array}$ & $\begin{array}{c}5.297 \\
(0.992)\end{array}$ & 0.717 & 0.999 & -23.038 & -22.594 & -20.236 \\
\hline
\end{tabular}

In the table (1) we compute the MLE of distributions parameters, the corresponding RMSE (given in parentheses), Kolmogorov-Smirnov (K.S) test statistic, AIC (Akaike Information Criterion), CAIC (the consistent Akaike Information Criterion) and BIC (Bayesian information criterion) for every distribution. We find from K.S test statistic that at the level of significant 0.01 we can not reject that the data fits all earlier 
distributions but it fits more the $\mathrm{Kw} \mathrm{Kw} W(\mathrm{a}, \mathrm{b}, \alpha, \beta, \theta, \lambda)$ distribution. We see that the $\mathrm{Kw} \mathrm{Kw} \mathrm{W}(\mathrm{a}, \mathrm{b}, \alpha, \beta, \theta, \lambda)$ distribution has the smallest KS, AIC, CAIC and BIC so the $\mathrm{Kw} \mathrm{Kw} W(\mathrm{a}, \mathrm{b}, \alpha, \beta, \theta, \lambda)$ distribution can be the best fitted distribution compared with other distributions.

Table 2: The log-likelihood function, The likelihood ratio test statistic and p-values

\begin{tabular}{|l|c|c|c|c|c|}
\hline \multicolumn{1}{|c|}{ The Model } & $\mathrm{H}_{0}$ & $\begin{array}{c}\ell \\
\text { (log likelihood })\end{array}$ & $\begin{array}{c}\Lambda \\
\text { (The likelihood ratio } \\
\text { test statistic) }\end{array}$ & $\begin{array}{c}\text { df } \\
\text { (degrees of } \\
\text { freedom) }\end{array}$ & P-value \\
\hline E Kw W $(a, \alpha, \beta, \theta, \lambda)$ & $\mathrm{b}=0$ & 41.842 & 14.42 & 1 & $1.462 \times 10^{-4}$ \\
\hline $\mathrm{KWW}(\alpha, \beta, \theta, \lambda)$ & $\mathrm{a}=0, \mathrm{~b}=0$ & 33.901 & 30.302 & 2 & $2.63 \times 10^{-7}$ \\
\hline $\mathrm{EG} \mathrm{W}(\mathrm{a}, \beta, \theta, \lambda)$ & $\alpha=0, \mathrm{~b}=0$ & 38.794 & 20.516 & 2 & $3.508 \times 10^{-5}$ \\
\hline $\mathrm{E} \mathrm{W}(\alpha, \theta, \lambda)$ & $\mathrm{a}=0, \mathrm{~b}=0, \beta=0$ & 15.695 & 66.714 & 3 & $2.154 \times 10^{-14}$ \\
\hline $\mathrm{W}(\theta, \lambda)$ & $\mathrm{a}=0, \mathrm{~b}=0, \alpha=0, \beta=0$ & 13.519 & 71.066 & 4 & $1.354 \times 10^{-14}$ \\
\hline
\end{tabular}

*Note that the log likelihood of the Kw Kw W $(a, b, \alpha, \beta, \theta, \lambda)=49.052$

In the table (2) and based on the likelihood ratio test, where the $\mathrm{Kw} \mathrm{Kw} W(\mathrm{a}, \mathrm{b}, \alpha, \beta, \theta$, $\lambda)$ distribution generalizes the $\mathrm{E} \mathrm{Kw} \mathrm{W}(\mathrm{a}, \alpha, \beta, \theta, \lambda)$ distribution, the $\mathrm{Kw} \mathrm{W}(\alpha, \beta, \theta, \lambda)$ distribution, the EG W (a, $\beta, \theta, \lambda)$ distribution, the $\mathrm{E} \mathrm{W}(\alpha, \theta, \lambda)$ distribution, the $\mathrm{E} \mathrm{W}(\alpha$, $\theta, \lambda)$ distribution and the $\mathrm{W}(\theta, \lambda)$ distribution, we find from the $\mathrm{p}$-values that we can reject all null hypotheses when the level of significant is 0.01

\section{Conclusions}

The $\mathrm{Kw} \mathrm{Kw} \mathrm{W} \mathrm{distribution} \mathrm{is} \mathrm{an} \mathrm{important} \mathrm{distribution} \mathrm{in} \mathrm{world} \mathrm{of} \mathrm{data} \mathrm{sets} \mathrm{because} \mathrm{of}$ its flexible properties and its generalization of some important distributions as the $\mathrm{E} \underline{\mathrm{KW}}$ $\mathrm{W}$ distribution, the $\mathrm{Kw} \mathrm{W}$ distribution, the $\mathrm{EG} \mathrm{W}$ distribution, the $\mathrm{E} \mathrm{W}$ distribution and the $\mathrm{W}$ distribution. We encourage researchers to do more researches and applications on the $\mathrm{Kw} \mathrm{Kw} \mathrm{W}$ in univariate and multivariate cases.

\section{Acknowledgements}

We thank anyone suggested an improved comments specially the referees who provided helpful suggestions for this paper.

\section{References}

1. A. P. Prudnikov, Yu. A. Brychkov and O. I. Marichev. Integrals and Series, Vol. 3, Gordon and Breach Science Publishers, Amsterdam, (1986).

2. A. S. Wahed, A general method of constructing extended families of distribution from an existing continuous class: Journal of Probability and Statistical Science. 4 (2006), 165-177.

3. C. B. Arnold, N. Balakrishnan, H. N. Nagaraja, A first course in order statistics: John Wiley \& Sons, Inc. New York, (1992). 
4. E. A. El-Sherpieny, M. A. Ahmed, On the Kumaraswamy Kumaraswamy distribution. International Journal of Basic and Applied Sciences. 3 (2014), 372-381.

5. G. M. Cordeiro and M. Castro. A new family of generalized distributions, Journal of Statistical Computation \& Simulation. 81 (2010), 1563-5163.

6. J. R. M. Hosking, L moments: analysis and estimation of distribution using linear combination of order statistic, Journal of the Royal Statistical Society. Series B, 52(1990), 105-124.

7. M. R. Leadbetter, G. Lindgren, H. Rootzen, Extremes and Related Properties of Random Sequences and Process, Springer Verlag, New York, 1987.

8. N. Eugene, C. Lee, and F. Famoye. The Beta-normal distribution and its applications, Communications in Statistics, Theory and Methods. 31(2002), 497-512.

9. R. M. Mahmoud, E. A. El-Sherpieny, M. A. Ahmed. The New Kumaraswamy Kumaraswamy Family of Generalized Distributions with Application, Pakistan Journal of Statistics and Operation Research.11(2015), 159-180.

10. W. Q. Meeker, L.A. Escobar. Statistical Methods for Reliability Data, John Wiley, New York, (1998). 